\title{
BCG vaccination induces HIV target cell activation in HIV-exposed infants in a randomized trial
}

\author{
Melanie A. Gasper, ${ }^{1,2}$ Anneke C. Hesseling, ${ }^{3}$ Isaac Mohar, ${ }^{2}$ Landon Myer, ${ }^{4}$ Tali Azenkot, ${ }^{2}$ \\ Jo-Ann S. Passmore, ${ }^{5,6}$ Willem Hanekom, ${ }^{7}$ Mark F. Cotton, ${ }^{3}$ I. Nicholas Crispe, ${ }^{1,2}$ Donald L. Sodora, ${ }^{1,2}$ \\ and Heather B. Jaspan, ${ }^{1,2,5}$ \\ 'University of Washington, Seattle, Washington, USA. ${ }^{2}$ Center for Infectious Disease Research, Seattle, Washington, USA. \\ ${ }^{3}$ Department of Paediatrics and Child Health, Faculty of Medicine and Health Sciences, Stellenbosch University, Tygerberg, \\ South Africa. ${ }^{4}$ Division of Epidemiology and Biostatistics, School of Public Health and Family Medicine, and ${ }^{5}$ Department \\ of Pathology, University of Cape Town, Cape Town, South Africa. ${ }^{6}$ National Health Laboratory Service, Cape Town, South \\ Africa. ${ }^{7}$ South African Tuberculosis Vaccine Initiative, Institute of Infectious Disease and Molecular Medicine, University of \\ Cape Town, Cape Town, South Africa.
}

BACKGROUND. Bacillus Calmette-Guérin (BCG) vaccine is administered at birth to protect infants against tuberculosis throughout Africa, where most perinatal HIV-1 transmission occurs. We examined whether BCG vaccination alters the levels of activated HIV target T cells in HIV-exposed South African infants.

METHODS. HIV-exposed infants were randomized to receive routine (at birth) or delayed (at 8 weeks) BCC vaccination. Activated and CCR5-expressing peripheral blood CD4+ $\mathrm{T}$ cell, monocyte, and NK cell frequencies were evaluated by flow cytometry and immune gene expression via PCR using Biomark (Fluidigm).

RESULTS. Of 149 infants randomized, 92\% $(n=137)$ were retained at 6 weeks: 71 in the routine BCC arm and 66 in the delayed arm. Routine BCC vaccination led to a 3-fold increase in systemic activation of HIV target $\mathrm{CD}^{+}{ }^{+} \mathrm{CCR} 5^{+} \mathrm{T}$ cells $\left(\mathrm{HLA}-\mathrm{DR}^{+} \mathrm{CD} 38^{+}\right)$at 6 weeks $(0.25 \%$ at birth versus $0.08 \%$ in delayed vaccination groups; $P=0.029$ ), which persisted until 8 weeks of age when the delayed arm was vaccinated. Vaccination of the infants in the delayed arm at 8 weeks resulted in a similar increase in activated CD4+CCR5 ${ }^{+} \mathrm{T}$ cells. The increase in activated $\mathrm{T}$ cells was associated with increased levels of MHC class II transactivator (CIITA), IL12RB1, and IFN- $\alpha 1$ transcripts within peripheral blood mononuclear cells but minimal changes in innate cells.

Role of funding source: The funder of the study had no role in study design, data collection, data analysis, data interpretation, or writing of the report. The corresponding author had full access to all the data in the study and had the final responsibility for the decision to submit for publication.

Authorship note: D.L. Sodora and H.B. Jaspan contributed equally to this work.

Conflict of interest: The authors have declared that no conflict of interest exists.

Submitted: November 28, 2016 Accepted: March 1, 2017 Published: April 6, 2017

Reference information: JCI Insight. 2017;2(7):e91963. https:// doi.org/10.1172/jii.insight.91963.
CONCLUSION. BCG vaccination induces immune changes in HIV-exposed infants, including an increase in the proportion of activated CCR5 ${ }^{+} \mathrm{CD} 4^{+}$HIV target cells. These findings provide insight into optimal BCG vaccine timing to minimize the risks of HIV transmissions to exposed infants while preserving potential benefits conferred by BCG vaccination.

TRIAL REGISTRATION. ClinicalTrials.gov NCT02062580.

FUNDING. This trial was sponsored by the Elizabeth Glaser Pediatric AIDS Foundation (MV-00-9900-01871-0-00) and the Thrasher Foundation (NR-0095); for details, see Acknowledgments.

\section{Introduction}

Despite the implementation of wide-scale prevention of mother-to-child transmission of HIV-1 (PMTCT) programs, perinatal HIV-1 transmission remains a significant contributor to the HIV epidemic. Nearly half of MTCT occurs during breastfeeding (1). Therefore, an understanding of factors influencing breastfeeding-associated MTCT is critical to eliminating pediatric HIV.

The live-attenuated bacillus Calmette-Guérin (BCG) vaccine is currently the only licensed tuberculosis (TB) vaccine; it is usually administered shortly after birth to protect against TB in childhood. BCG vaccine is administered to over 100 million children worldwide annually (2), including in sub-Saharan Africa, 
where the majority of MTCT occurs (3). In HIV-unexposed children, BCG vaccination is safe, efficacious, and cost-effective against TB, particularly cases of disseminated disease (2). However, studies assessing BCG immunogenicity in $\mathrm{HIV}^{-}(\mathrm{HEU})$ infants demonstrate altered cellular responses to BCG and other vaccines $(4,5)$, suggesting that HEU infants might not gain the same benefits from BCG vaccination as infants that are not HIV exposed.

In addition to altered immunogenicity in HEU infants, the immune activation induced by BCG vaccination has the potential to render these neonates more susceptible to HIV infection (6). HIV preferentially infects and replicates in activated $\mathrm{CD}^{+} \mathrm{T}$ cells $(7,8)$. Further, peripheral blood mononuclear cells (PBMCs) from persons with chronic $\mathrm{CD}^{+} \mathrm{T}$ cell activation are more susceptible to in vitro infection with HIV (8). In contrast, a reduced state of CD4 T cell immune activation is associated with protection against HIV infection both in vivo and in vitro. Highly exposed, persistently seronegative individuals have relatively lower levels of $\mathrm{CD}^{+} \mathrm{T}$ cells coexpressing the activation markers CD38 and HLA-DR, compared with persons who become infected with HIV (9). Additionally, because mucosally transmitted HIV is almost exclusively CCR 5 tropic, activated $\mathrm{CD} 4{ }^{+}$target cells expressing the coreceptor CCR 5 are even more likely to become infected. Indeed, low levels of peripheral and mucosal CCR5-expressing CD4 ${ }^{+} \mathrm{T}$ cells are associated with a lack of simian immunodeficiency virus (SIV) acquisition in infant sooty mangabeys following low-dose oral challenge (10). These results contrast with the pathogenic SIV infection of rhesus macaques, which have higher frequencies of CCR $5^{+} \mathrm{CD} 4^{+} \mathrm{T}$ cells and higher rates of vertical SIV transmission (10). Together, these data suggest that $\mathrm{CD} 4^{+} \mathrm{CCR} 5^{+} \mathrm{T}$ cell activation contributes to HIV/SIV susceptibility and acquisition. Importantly, BCG vaccination of infant macaques has been associated with persistent CD4 T cell activation (6). Finally, BCG-vaccinated infant macaques generally required fewer SIV exposures to successfully initiate an SIV infection following low-dose oral exposure (6).

The beneficial aspects of BCG vaccination may go beyond TB prevention, as it is also reportedly associated with lower all-cause infant mortality (reviewed in ref. 11). Although there is a paucity of randomized trial data (11) and there is no confirmed biological mechanism to support this observation, the recent discovery of BCG vaccination-induced posttranslational histone modifications in adult innate immune cells, which are associated with more robust responses to heterologous antigens (termed "trained innate immunity"), provides one mechanism by which these nonspecific benefits may occur (12). Due to the possible association of BCG with health in HIV-unexposed infants, it may be important that BCG is retained in infant vaccine schedules or that novel TB vaccine candidates preserve these benefits conferred by BCG. However, the potential for BCG and related vaccinations to induce in vivo CD4 ${ }^{+} \mathrm{T}$ cell activation and influence HIV susceptibility in HIV-exposed infants has yet to be explored.

Considering the high risk of HIV and TB infection in HIV-exposed infants in sub-Saharan Africa and the widespread use of BCG in HIV-endemic areas, it is crucial to explore the potential for BCG vaccination to induce in vivo $\mathrm{CD}^{+} \mathrm{T}$ cell activation and influence HIV susceptibility in these populations. To this end, we performed a randomized clinical trial of routine (at birth) versus delayed (administered at 8 weeks of age) BCG vaccination in HIV-exposed South African infants. Our goal was to evaluate the effect of BCG on activation of $\mathrm{CD} 4^{+} \mathrm{T}$ cells and innate monocytes and NK cells. We demonstrate for the first time to our knowledge that BCG vaccination at birth causes a sustained increase in frequencies of activated $\mathrm{CD}^{+} \mathrm{CCR} 5^{+} \mathrm{HIV}$ target cells in HIV-exposed infants, which persists until at least 8 weeks of age and, therefore, has the potential to increase HIV acquisition in this vulnerable population that is repeatedly exposed to HIV via breast milk. Our results highlight the importance of further vaccine studies in HIV-exposed infants. These should aim to carefully evaluate the specific (protection against TB) and the nonspecific beneficial effects conferred by BCG vaccination as well as its potential for increasing $\mathrm{T}$ cell activation associated with perinatal HIV transmission.

\section{Results}

Cohort. Between June 22, 2010, and November 31, 2011, 178 mother-infant pairs were screened for eligibility (Figure 1). Of these infants, 149 were enrolled and 29 were excluded from participation. Reasons for exclusion included maternal refusal to participate ( $n=26 ; 15 \%$ of total assessed), receipt of BCG vaccination prior to randomization $(n=2 ; 1 \%)$, and not meeting study inclusion criteria $(n=1 ;<1 \%$; Figure 1$)$.

Of the 149 infants ultimately enrolled, $78(52 \%)$ were randomized to receive routine BCG vaccination and 71 (48\%) were randomized to receive delayed vaccination (Figure 1). Median maternal CD4 count of the enrolled cohort was 362 cells $/ \mathrm{mm}^{3}$ (interquartile range [IQR] 253-508 cells $/ \mathrm{mm}^{3}$ ). The majority of mothers 


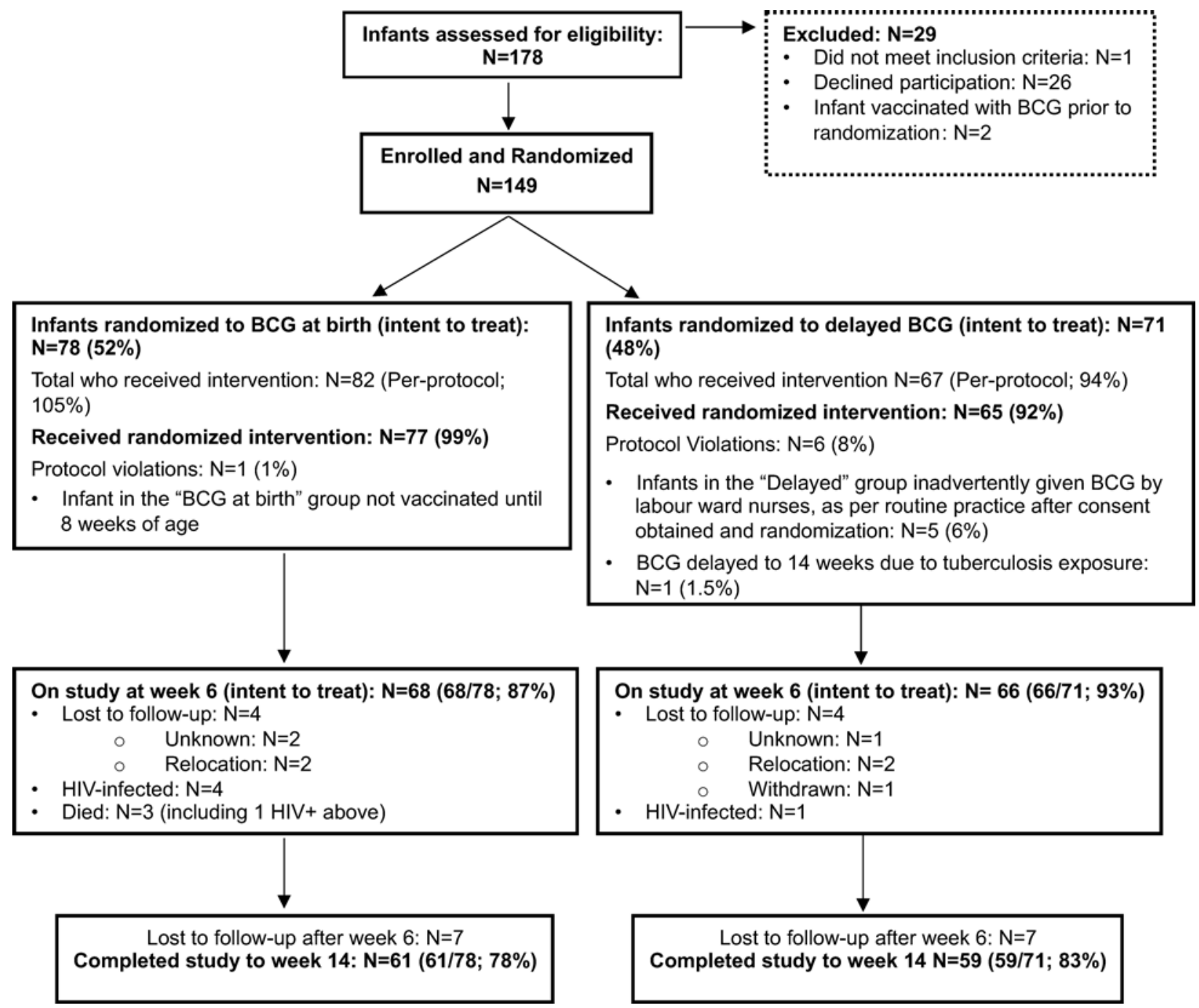

Figure 1. Consort diagram of the intent-to-treat population.

received only zidovudine, with single-dose nevirapine for prevention of MTCT ( $n=80,55 \%)$; the remainder received combination antiretroviral (cART). The median birth weight of the infants was 3,100 g (IQR 2,800-3,320 g), and median gestational age was 39 weeks (IQR 38-40 weeks); 82 infants (55\%) were female. Baseline characteristics of all randomized infants and their mothers by trial arm are found in Table 1.

Five infants who were randomized to the delayed arm were inadvertently vaccinated at birth by the routine labor ward staff prior to discharge, and one infant randomized to birth BCG was vaccinated at 8 weeks. Six infants tested HIV DNA PCR positive; 4 of $78(5.1 \%)$ in the routine arm and 1 of $71(1.4 \%)$ in the delayed arm (Figure 1). These infants were excluded from downstream immunological analyses and referred for immediate initiation of cART therapy. There were 3 infant deaths, all in the routine arm (i.e., 3.9\% mortality in the routine arm and $0 \%$ in the delayed arm), one of whom was also HIV infected. An additional 22 infants were lost to follow-up, such that at 6 and 14 weeks of age, 134 and 120 infants ( $90 \%$ and $83 \%$, respectively) were retained in the intention to treat immunological analysis. There were no remarkable differences in baseline characteristics between those retained in the trial and those lost to follow-up by 6 weeks of age (Table 1). There were 137 other adverse events: 72 in the routine arm and 65 in the delayed arm (i.e., some infants had more than one adverse event; Supplemental Table 2; supplemental material available online with this article; https://doi.org/10.1172/jci.insight.91963DS1). There were no statistically significant differences in frequency of serious adverse events between the routine and delayed arms, including $(P=0.135)$ or excluding $(P=0.459)$ HIV infections. All HIV infections except one were assumed all to have occurred in utero (i.e., HIV DNA PCR positive within 48 hours of life, ref. 13). 
Table 1. Baseline characteristics of all infants and mothers and those retained at 6 weeks in the intent-to-treat population

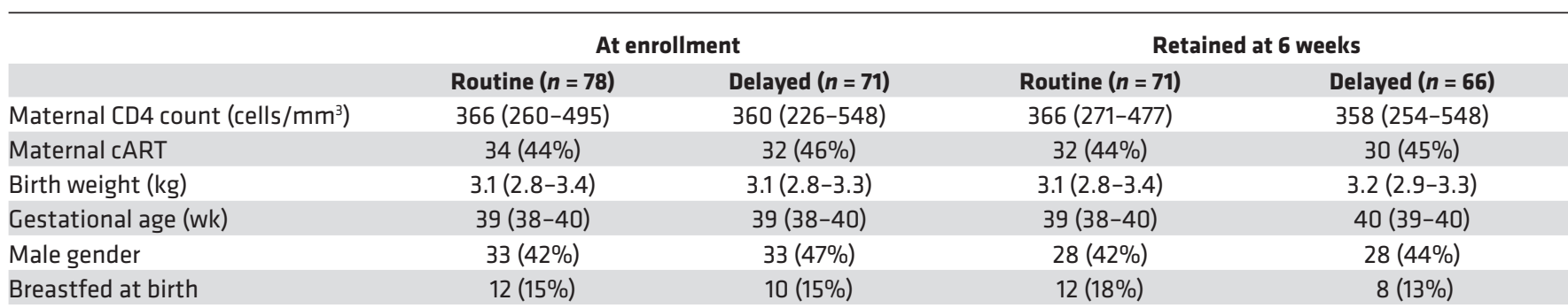

Data are $n(\%)$ or median (IQR).

Likewise, there was no difference in infectious morbidities, including pneumonia, meningitis, cough, chest infection, abscess, and gastroenteritis ( $n=20$ in the routine arm and $n=19$ in the delayed arm, $P>0.999$ ). No adverse event was deemed related to study intervention or participation.

$B C G$-vaccinated infants have significantly higher $C D 4^{+} T$ cell activation. $\mathrm{CD} 4^{+} \mathrm{T}$ cell activation is associated with increased risk of HIV infection and disease progression $(7,14)$. We therefore evaluated $\mathrm{T}$ cell activation in peripheral blood from birth through the end of follow-up in infants randomized to birth versus delayed BCG vaccination via flow cytometric detection of HLA-DR, CD38, and Ki67 (Figure 2A). At birth, there was no significant difference in the proportion of HLA-DR expression on $\mathrm{CD} 4^{+}$or CD8 ${ }^{+}$ $\mathrm{T}$ cells. By 2 weeks of age, a slight increase in $\mathrm{CD}^{+} \mathrm{T}$ cell activation was seen in the birth-vaccinated arm compared with the delayed BCG arm, but this was not significant (median CD4 ${ }^{+} \mathrm{HLA}_{\mathrm{D}} \mathrm{DR}^{+}$cells $1.1 \%$, IQR $0.68 \%-1.6 \%$ for the birth BCG-vaccinated arm, and median CD $4^{+} \mathrm{HLA}-\mathrm{DR}^{+}$cells $0.94 \%$, IQR $0.69 \%-1.4 \%$ in the delayed vaccination arm; $P=0.106$ ). By 6 weeks of age, the infants who had received routine $B C G$ vaccination, however, had significantly higher levels of $\mathrm{CD}^{+} \mathrm{T}$ cells expressing the activation marker HLA-DR compared with those in the delayed arm who had not yet received BCG vaccination (median $1.8 \%$, IQR $1.0 \%-2.8 \%$ versus median $1.3 \%$, IQR $0.81 \%-2.4 \% ; P=0.028$; Figure 2B). This difference was not evident in $\mathrm{CD}^{+} \mathrm{T}$ cells (Figure $2 \mathrm{C}$ ).

CCR5 is highly expressed on activated HLA-DR ${ }^{+} \mathrm{CD} 38^{+}$cells in adults and serves as the major coreceptor for transmitted HIV variants (15). We therefore compared CCR5 and activation marker coexpression on $\mathrm{CD}^{+} \mathrm{T}$ cells between the two arms. As with CD4 ${ }^{+} \mathrm{HLA}-\mathrm{DR}{ }^{+}$cells, the percentage of CCR5expressing, highly activated $\mathrm{CD} 4^{+} \mathrm{CD} 38^{+} \mathrm{HLA}-\mathrm{DR}{ }^{+} \mathrm{T}$ cells was similar at baseline and not significantly different at 2 weeks of age (Figure 3A). By 6 weeks of age, this cell subset was 3.3-fold higher in the infants who had received BCG vaccination at birth (median $0.253 \%$ in the birth-vaccinated arm versus $0.075 \%$ in the delayed, i.e., unvaccinated, arm; $P=0.029$; Figure 3A). Although both arms displayed an increase in $\mathrm{CD}^{+} \mathrm{T}$ cell activation at between 6 weeks and 8 weeks of age (infants also received their first doses of rotavirus; hepatitis B; inactivated polio; Haemophilus influenzae type b; diphtheria, tetanus, acellular pertussis [DTaP], and conjugated pneumococcal vaccinations, as well as their second dose of oral polio vaccine, at 6 weeks), significantly higher $\mathrm{CD}^{+} \mathrm{T}$ cell activation persisted in the routine BCG arm at 8 weeks of age. As this was the final time point at which the delayed group had not yet received BCG, this suggests that BCG is the major driver of $\mathrm{T}$ cell activation. In a generalized estimating equation population-averaged model, BCG vaccination significantly increased the rate of rise in $\mathrm{CD} 4^{+} \mathrm{CCR} 5^{+} \mathrm{CD} 38^{+} \mathrm{HLA}-\mathrm{DR}{ }^{+}$cell frequency that occurs through time until 8 weeks, whether before (coefficient $0.18,95 \%$ CI $0.09-0.27 ; P<$ 0.0001 ) or after adjusting for maternal CD4 count and gestational age (coefficient $0.18,95 \%$ CI $0.08-0.27$, $P<0.0001$ ). Age in weeks was also independently associated with increased $\mathrm{T}$ cell activation but to a lesser extent than BCG vaccination (coefficient 0.02 , 95\% CI 0.007-0.03). There were no differences in $\mathrm{CD}^{+} \mathrm{T}$ cell CCR5 and activation marker expression at any time point (Figure 2C and data not shown).

While infants from the routine vaccination arm maintained a similar, elevated level of $\mathrm{CD} 4^{+} \mathrm{T}$ cell activation between the 8 - and 14 -week time points, infants from the delayed arm, who were vaccinated at 8 weeks, had an increase in their $\mathrm{CD}^{+} \mathrm{T}$ cell activation profile during this time, effectively diminishing the difference between the groups at 14 weeks (Figure 3A). Furthermore, the increase in $\mathrm{CD}^{+} \mathrm{T}$ cell activation between birth and 6 weeks after conventional vaccination (Figure 3B, left; median $0.03 \%$ at birth versus $0.25 \%$ at week $6 ; P<0.0001$ ) was similar to that between delayed vaccination and 6 weeks 
A

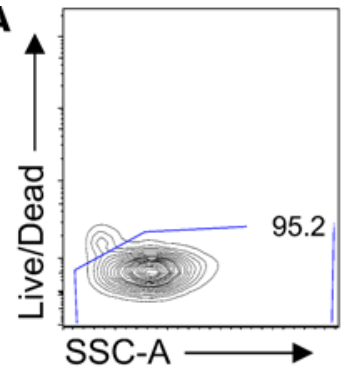

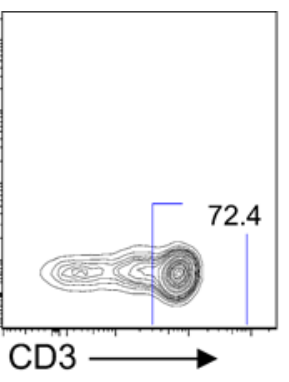
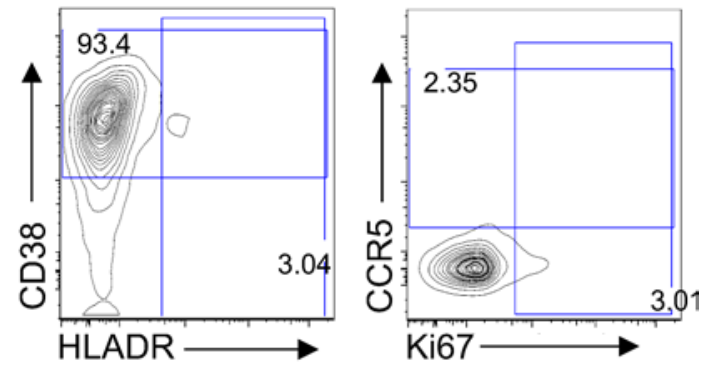

B

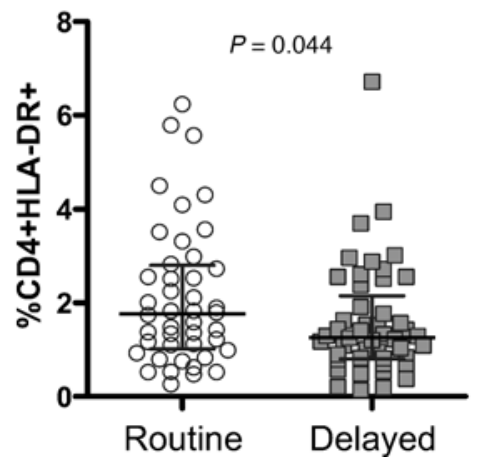

C

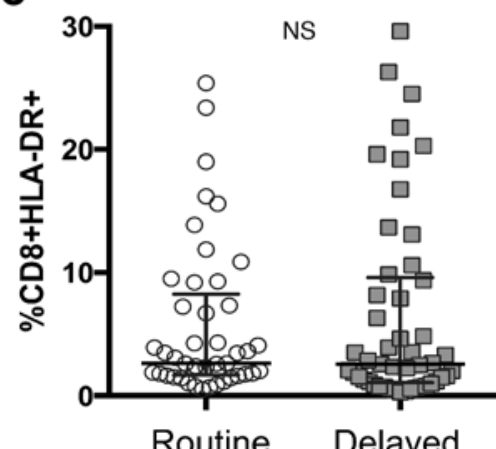

Figure 2. BCG vaccination of HIV-exposed infants is associated with an increase in activated CD4+ $\mathbf{T}$ cells. (A) Representative flow plot depicting gating strategy for determining the frequency of CD4 $4^{+} \mathrm{HLADR}^{+}$cells of total (B) $\mathrm{CD} 4^{+}$and (C) $\mathrm{CD} 8^{+}$T cells in routine (circles; $n=45$ ) or delayed (squares; $n=49$ ) BCG-vaccinated infants at 6 weeks of age, when only the "routine" arm had received vaccination, while the "delayed" arm was unvaccinated. Only samples with >1,000 live $\mathrm{CD}^{+}$cells were included in the analysis. Lines depict medians; whiskers depict interquartile ranges.

after delayed vaccination (Figure 3B, right; median $0.14 \%$ at week 8 vs. $0.41 \%$ week $14 ; P=0.0008$ ). Thus, a similar increase in $\mathrm{CD} 4^{+} \mathrm{T}$ cell activation occurred whether infants were BCG vaccinated at birth or later in infancy.

PBMC gene expression analysis. BCG vaccination of adults has been described to induce a primed innate immune system, previously termed trained innate immunity, which persists for up to 1 year after vaccination (12). To measure differences in global or innate immune activation that might accompany the $\mathrm{CD} 4^{+} \mathrm{T}$ cell activation we identified, we undertook microfluidic high-throughput quantitative RT-PCR (Fluidigm) on PBMCs 2 weeks after the routine (at birth) BCG group had been vaccinated (Figure 4). Infants vaccinated with BCG at birth displayed statistically higher transcript levels of the MHC class II transactivator (CIITA) $(P=0.019)$ and lower levels of CCL-2 $(P=0.033)$ and IL-1 $\alpha(P=0.025$; Figure 4 , A and B), although these differences did not maintain statistical significance after adjustment for multiple comparisons. BCG-vaccinated infants also displayed a trend toward higher levels of IL-18 and CCL5 mRNA and lower levels of IL-10 and IL-6 mRNA, although these were not significantly different $(P<0.1$ for all; Figure 4A). Regression analysis that included infants from both groups showed a positive relationship between percentage of $\mathrm{CD}^{+} \mathrm{CCR} 5^{+} \mathrm{HLA}-\mathrm{DR}{ }^{+} \mathrm{CD} 38^{+} \mathrm{T}$ cells at 6 weeks and the transcript levels of inflammatory genes, including CIITA $(P=0.019)$, IFN- $\alpha(P=0.016)$, and IL12RB1 $(P=0.038)$, and an inverse relationship between $\mathrm{CD} 4^{+} \mathrm{T}$ cell activation and IL-1 $\alpha(P=0.029)$ (Figure $\left.4 \mathrm{C}\right)$. In addition, CD8a transcript levels were positively related to $\mathrm{CD} 4^{+} \mathrm{CCR} 5^{+} \mathrm{HLA}-\mathrm{DR}{ }^{+} \mathrm{CD} 38^{+} \mathrm{T}$ cells $(P=0.001)$.

Innate cell phenotypic distribution and activation. Both monocytes and NK cells display properties of BCGmediated innate immune "training," in which epigenetic reprogramming of cells results in nonspecific protection from infections $(12,16)$. Thus, to evaluate the potential for innate immune training and/or activation and because monocyte and NK cell phenotypes associate with disparate cellular function and activation, we assessed the phenotypic distribution of these cells at 8 weeks of age ( 8 weeks after BCG vaccination in the routine arm, but prior to BCG vaccination of the delayed arm). We also measured expression of PD-1 and Tim-3 downregulation, both of which indicate activated cellular subsets of monocytes and NK cells (17-19). We investigated the ex vivo phenotypic distribution of $\mathrm{CD} 16^{+} \mathrm{CD} 56^{-}$(anergic or progenitor), $\mathrm{CD} 16^{+} \mathrm{CD} 56^{+}$ (cytotoxic), and CD56 $6^{\text {bright }}$ (cytokine producing) NK cells and NK cell activation status (through HLA-DR, 
A

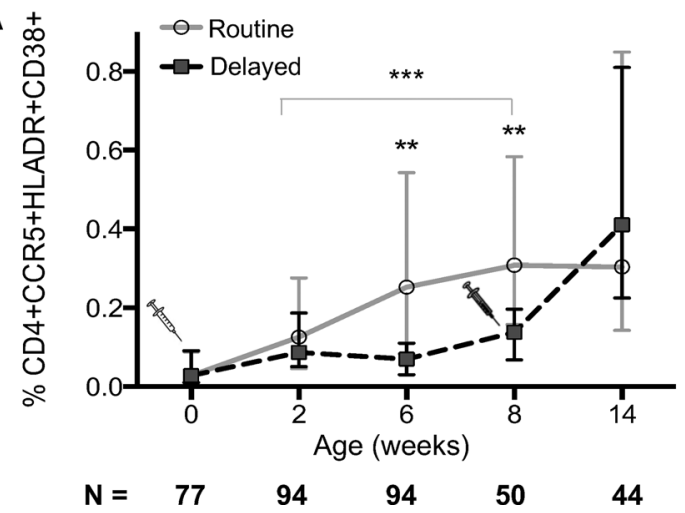

B

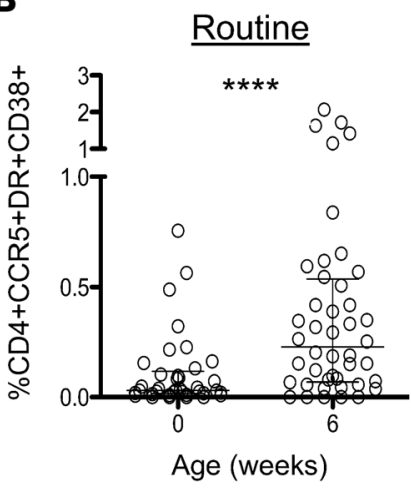

Delayed

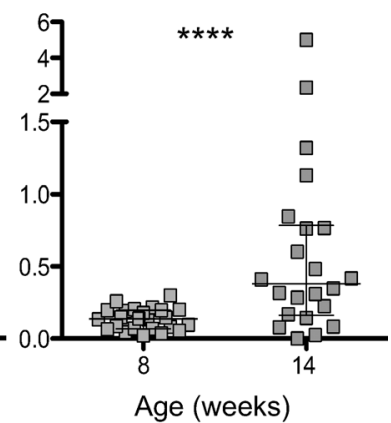

Figure 3. BCG vaccination of HIV-exposed infants is associated with an increase of activated CD4+CCR5+ HIV target cells. (A) Longitudinal frequency of activated $\mathrm{CD}^{+}{ }^{+} \mathrm{CCR} 5^{+} \mathrm{HLA}-\mathrm{DR}{ }^{+} \mathrm{CD} 38^{+} \mathrm{T}$ cells from birth through 14 weeks in routine (gray circles) versus delayed (black squares) BCG-vaccinated infants. Syringe graphics indicate the time of vaccination in the routine arm (gray) or the delayed arm (black). (B) Plots depicting the increase in activated CCR5 ${ }^{+}$CD4 $4^{+}$target cells in the routine or delayed groups 6 weeks after vaccination in each group (routine: $n=37$ at 0 weeks, $n=44$ at 6 weeks; delayed: $n=24$ at 8 weeks, $n=21$ at 14 weeks; only samples with $>1,000$ live $\mathrm{CD}^{+}$cells were included in the analysis. Lines depict the medians; whiskers represent the interquartile ranges. ${ }^{* *} P<0.01 ;{ }^{* *} P<0.001 ;{ }^{* * *} P<0.0001$.

PD-1, and Tim-3 expression). We found significantly higher frequencies of CD56 $6^{\text {bright }}\left(\mathrm{CD} 56^{++}\right)$NK cells expressing PD-1 in the vaccinated (routine) infants compared with those in the delayed arm who had not yet been vaccinated $(0.76 \%$ versus $0.40 \% ; P=0.045$; Figure $5 \mathrm{D})$, although these differences did not persist after adjustment for multiple comparisons. No other differences in NK cell phenotype and/or activation status were evident between routine (early) and delayed BCG-vaccinated infants at 8 weeks (Figure 5). When assessing monocytes, we found no differences in ex vivo monocyte phenotype (as measured via CD14 and/ or CD16 [Fc $\gamma$ III receptor] expression via flow cytometry; Supplemental Figure 1, A and B), or in activation (as measured by the concentration of plasma sCD163; Supplemental Figure 1C), or in the expression of Tim-3 or PD-1 (data not shown). Additionally, no association was found between the percentage of CD16 monocytes or plasma sCD163 and $\mathrm{CD} 4^{+} \mathrm{CCR} 5^{+} \mathrm{HLADR}{ }^{+} \mathrm{CD} 38^{+} \mathrm{T}$ cells (data not shown).

Plasma inflammatory cytokine concentrations. Low levels of inflammatory cytokines are associated with low risk of HIV infection (20). We therefore assessed cytokine levels in plasma of infants randomized to the routine versus delayed BCG arms at 2 and 6 weeks of age using multiplex bead array (Luminex). We found no significant differences in log-transformed plasma levels of GMCSF, IFN- $\alpha$, IFN- $\gamma$, IP-10, MCP1, TNF- $\alpha$, or IL-8 at week $2(n=58)$ or week $6(n=62)$ (Supplemental Figure 2$)$. MIP-1 $\beta$ plasma concentration was elevated in the delayed BCG arm at 6 weeks $(P=0.039)$, although this difference was not significant after adjusting for multiple comparisons.

\section{Discussion}

Although cART therapy during pregnancy and breastfeeding has greatly reduced MTCT in most HIV endemic countries, transmission still occurs, predominantly due to suboptimal implementation of antiretroviral prophylaxis, resulting in 150,000 new pediatric infections annually (1). Further, in many settings in which HIV is prevalent, BCG vaccination is administered shortly after birth to protect against early childhood TB. Importantly, this often occurs before definitive HIV testing can take place. Therefore, studies such as the one described here are critical in discerning the risks and benefits associated with BCG (or other) vaccinations.

Although pathogen-specific immune activation is critical to fighting infection, the timing, magnitude, and type of activated cells are all important factors in guiding a nuanced immune response that determines disease outcome. $\mathrm{CD}^{+} \mathrm{T}$ cell activation, in particular, has recently been implicated in increased TB disease risk in two separate populations, including BCG-vaccinated infants and TB-infected adolescents (21). Moreover, influenza disease severity is exacerbated by excessive $\mathrm{CD}^{+} \mathrm{T}$ cell cytokine response to viral antigen (22). Mounting evidence suggests that T cell "quiescence," or a low level of T cell activation, is associated with protection against HIV infection $(8,9)$. Conversely, cord blood lymphocyte activation caused by maternal parasitic infections correlates with increased HIV MTCT (23). Here, we observed a change in the percentage of total activated $\mathrm{T}$ cells in blood following BCG vaccination that may be clinically relevant in 
A

B
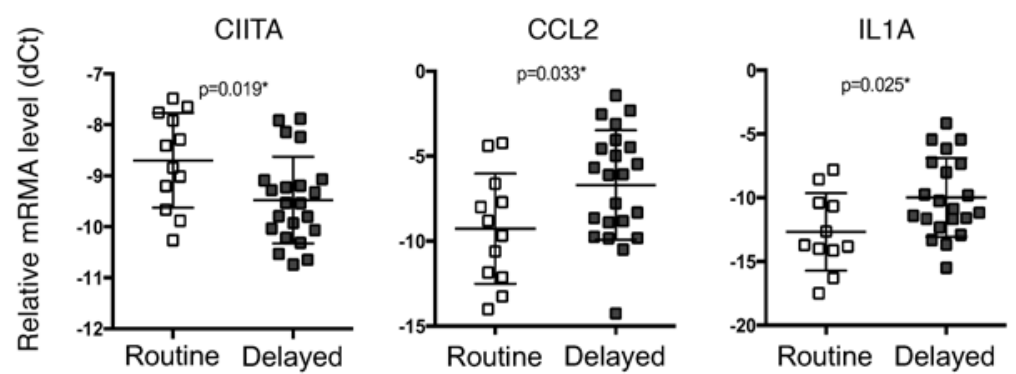

Relative expression

(dCt):

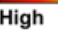

Low
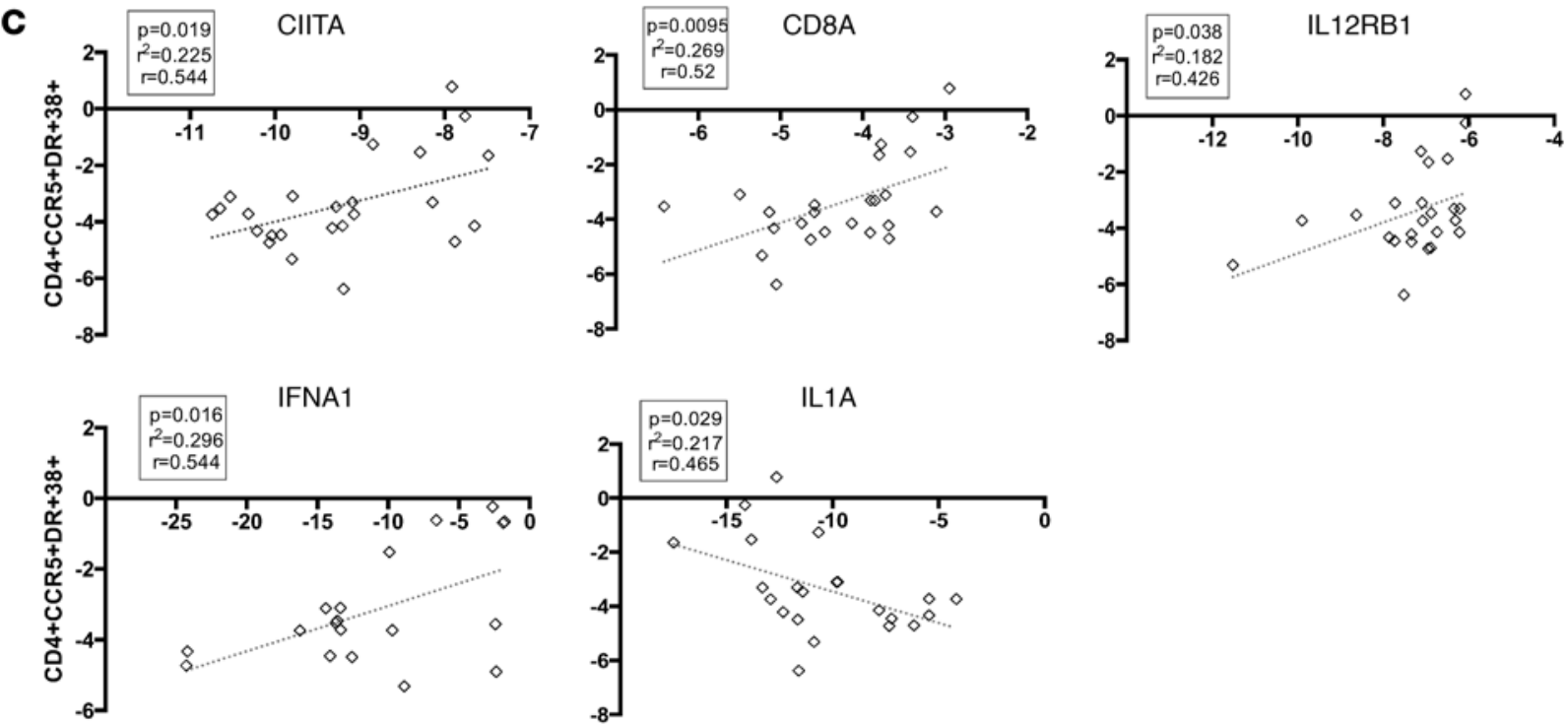

Figure 4. Fluidigm immune gene expression analysis in routine and delayed BCG-vaccinated HIV-exposed infants at 2 weeks of age. Expression of 48 genes (including 2 housekeeping genes) was measured via Biomark (Fluidigm) quantitative real-time PCR analysis at 2 weeks of age when only the "routine" arm had received vaccination. (A) Fold change of gene expression between the routine versus delayed BCG-vaccinated groups. (B) - $\Delta$ Ct (calculated by $\mathrm{Ct}_{\text {gene of interest }}-\mathrm{Ct}_{\mathrm{CAPDH}}$ ) of genes that demonstrated significantly differential expression between the groups. Lines depict the median; whiskers depict interquartile range. (C) Linear regression of gene expression of CIITA, CD8A, IL12RB1, IFNA1, and IL1A as determined at 2 weeks of age (-dCt expression value is shown on the $x$ axis) and subsequent activation of activated CD4+CCR5 ${ }^{+}$HLA-DR+CD38+ HIV target cells at 6 weeks in all infants combined (shown on the $y$ axis).

the context of HIV exposure. In HIV-exposed adults, differences in CD4 ${ }^{+} \mathrm{CD} 38^{+} \mathrm{HLA}-\mathrm{DR}{ }^{+} \mathrm{T}$ cell frequencies of less than $1 \%$, representing only 1.3 - to 1.5 -fold higher target cell frequencies, exist between those that later become infected and those that remain HIV uninfected (9). The difference we report in systemic $\mathrm{T}$ cell activation here represents, on average, a greater than 3 -fold increase in activation in infants who received BCG vaccination compared with those that had not yet been vaccinated at 6 weeks of age. Further, expression of the HIV coreceptor CCR5 was included in our analysis, which may impart an even greater susceptibility of these cells to HIV infection. In support of this, in SIV-exposed infant nonhuman primates, the availability of HIV target cells is a key factor in determining risk for breast milk SIV transmission (10). Most importantly, a recent study of intradermal BCG vaccination of infant macaques provides evidence for BCG-induced increased susceptibility to repeated low-dose oral SIV exposure (6), which mimics exposure via breastfeeding. Indeed, BCG vaccination was associated with an increase in $\mathrm{T}$ cell activation in blood, as well as in the oral and gastrointestinal tissue, known sites of viral entry during oral HIV/SIV exposure $(6,24)$. Additionally, the sustained T cell activation evident in the systemic circulation of BCG-vaccinated infants in our study suggests that BCG may create a prolonged window of increased HIV susceptibility in 


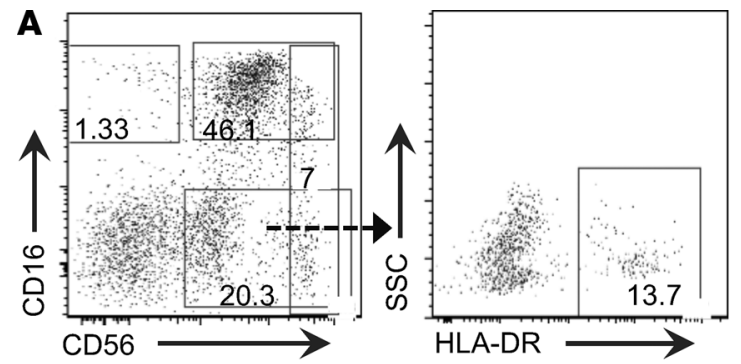

C

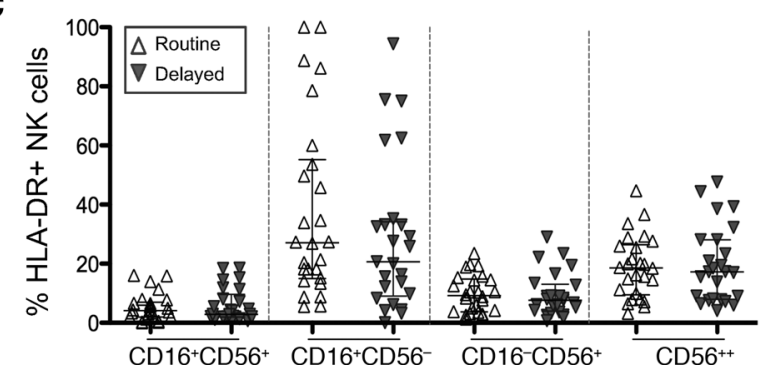

B

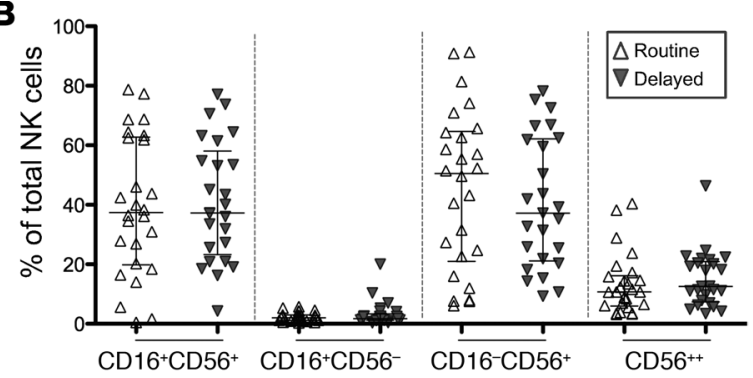

D

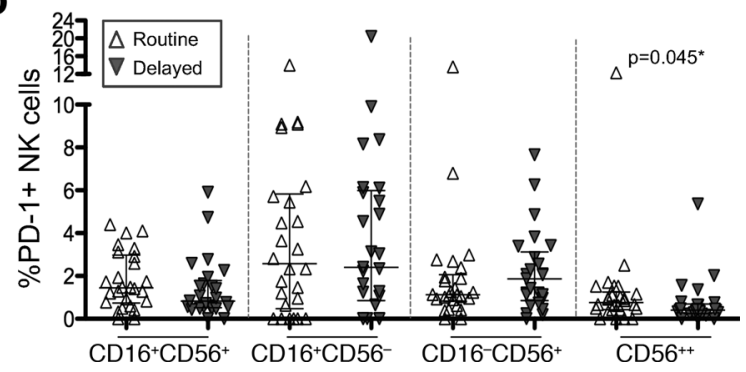

Figure 5. NK cell phenotype and activation in routine and delayed BCG-vaccinated HIV-exposed infants at 8 weeks of age. (A) Representative flow plot depicting $\mathrm{CD}^{+} 6^{+}$and $\mathrm{CD}^{-} 6^{+} \mathrm{NK}$ cells in PBMCs, which were previously gated on singlets, live, and Lin populations (CD3-CD19-CD14-). (B) Graphs depicting the overall NK cell phenotypic distribution in routine (white triangles; $n=26$ ) or delayed (gray inverted triangles; $n=25$ ) BCG-vaccinated infants at 8 weeks of age and (C) activation status of each subset, as measured by the percentage of HLA-DR-expressing NK cells. Only infants in the routine arm received vaccination at this point, while the delayed arm was unvaccinated. Lines depict the median of each group; whiskers represent the interquartile range. Statistics were generated using a Mann-Whitney test for nonparametric data.

HIV-exposed human infants. The potential consequences of these immunological effects must be weighed against the high risk of TB early in life in HIV-exposed infants, with incidence rates of 41 per 1,000 child years (95\% CI 31-52 per 1,000 child years) among HIV-uninfected, exposed South African children (25).

In addition to the potential for increased susceptibility to HIV, increases in $\mathrm{T}$ cell activation can accelerate HIV disease progression. Intravenously administered BCG drives T cell activation, resulting in an associated increased in SIV viral load and more rapid disease progression in macaques $(26,27)$. Although in these studies BCG was administered intravenously, which contrasts standard clinical intradermal vaccination procedures, they provide proof of principle that $\mathrm{BCG}$ activation of $\mathrm{T}$ cells can lead to cellular susceptibility to SIV/HIV and, therefore, accelerate disease pathogenesis. Indeed, intradermally BCG-vaccinated infant macaques that became SIV infected demonstrated a significantly higher viral load than unvaccinated controls during the acute phase of infection (6). In humans, other infections, such as CMV and TB, hasten HIV disease progression in HIV-1-infected adults (28). Additionally, some vaccinations, such as pneumococcal polysaccharide vaccines, can cause transient immune activation-associated viral load increases $(29,30)$. In contrast to these vaccines, which have proven efficacy during HIV infection $(31,32)$, the benefits of BCG vaccination are not yet clear in HIV-infected infants, in whom there is also an increased risk of BCG immune reconstitution inflammatory syndrome and disseminated BCG disease in the absence of early cART initiation (33-35).

BCG vaccination induces nonspecific protection from infections through epigenetic reprogramming of monocytes in adult volunteers, termed trained innate immunity (12). The increased ability of innate and adaptive immune cells to produce cytokines in response to in vitro stimulation after BCG vaccination in adults is sustained for up to 1 year after vaccination. Whether trained innate immunity occurs in infants is not currently known. Observational data and one randomized clinical trial in HIV-unexposed infants suggest that BCG may improve all-cause morbidity and mortality in West African infants (36-38), but this benefit has not been assessed in high HIV prevalence settings, and systematic reviews of the literature have not shown any clear beneficial nonspecific effects associated with BCG vaccination, which may be setting specific (39). Although Jensen et al. demonstrated higher cytokine responses to heterologous antigens in BCG-vaccinated low-birthweight infants (40), these infants had additional comorbidities associated with being either small for gestational age or premature. Using unstimulated PBMCs, we found significantly higher transcript levels of CIITA, which acts as a positive regulator of class II MHC gene transcription, in BCG-vaccinated infants at 2 weeks of age. 
Because MHC class II molecules are primarily expressed on professional antigen-presenting cells, this may be indicative of early phagocyte activation. However, we found no further difference in monocyte phenotype or activation, as measured by plasma soluble CD163 levels, between routine and delayed BCG-vaccinated infants at 8 weeks of age (i.e., before the delayed group received their vaccination). Because monocytes play a major role in the response to Mycobacteria spp (reviewed in ref. 41) and because monocyte activation has the potential to activate $\mathrm{CD}^{+} \mathrm{T}$ cells, we hypothesized that increased monocyte activation in routine BCG-vaccinated infants compared with unvaccinated (delayed) controls would be apparent. However, differences in monocyte activation were not detected at 8 weeks of age between the routine and delayed BCG arms. This result was surprising since monocytes are known responders to Mycobacteria and display activation following BCG vaccination of infant macaques (6). It is possible that the increase in monocyte activation is subtle (and therefore we were unable to detect any with the sample size or the markers used) or is transient and not observable at the time points that we examined. Finally, a lack of detectable innate responses may be a reflection of the impaired vaccine-specific cellular responses previously described in HEU infants $(4,5)$. Interestingly, CIITA expression has been shown to enhance viral attachment to $\mathrm{CD} 4^{+}$cells, independent of HLA-DR expression (42), providing another potential route for BCG vaccine-induced increase in HIV susceptibility. We found a higher frequency of PD-1-expressing CD56 ${ }^{\text {bright }} \mathrm{NK}$ cells, known for their ability to produce cytokines (43), in routinely vaccinated infants compared with those who had not yet been vaccinated. Increased PD-1 expression on $\mathrm{CD} 56^{\text {bright }} \mathrm{NK}$ cells is known to occur following IFN- $\gamma$ production in response to mycobacterial stimulation (18). In this setting, increased PD-1 expression on CD56 ${ }^{\text {bright }} \mathrm{NK}$ cells 8 weeks after BCG vaccination may be indicative of NK cell-trained innate immunity (16). Further studies that more thoroughly evaluate innate immune cell changes, including epigenetics and/or RNAseq, as well as a long-term follow-up on the cohort will enable a full assessment of BCG-mediated trained innate immunity in HIV-exposed infants.

While infant vaccinations (including BCG) are a critical component of disease prevention, the benefits of vaccinating HIV-exposed babies should be carefully weighed in the context of the risk of increasing vertical transmission and increased risk of exposure to other pathogens. Although cART prophylaxis has successfully reduced rates of vertical transmission in many areas, our findings are particularly relevant in two scenarios that are commonly observed in developing countries: (a) instances of suboptimal implementation of cART therapy and (b) for women who seroconvert during pregnancy or early postpartum. In these situations, delaying BCG vaccination could decrease the window of HIV vulnerability as well as allow more time for maternal viral control. In conclusion, our findings provide impetus for further evaluating the timing of standard infant vaccinations in HIV-exposed infants to identify both the nonspecific benefits as well as immunologic changes that may affect HIV transmission. These data also provide valuable immunological insights to inform the development of TB and HIV vaccines that are based on recombinant or attenuated mycobacteria.

\section{Methods}

Study design. This was a randomized, open-label trial, performed at a primary care midwife obstetric unit in Khayelitsha, Western Cape Province, South Africa, between June 2010 and December 2011.

Participants. Eligibility requirements included mothers (legal guardian) willing and able to provide informed consent, confirmed maternal HIV infection, gestational age $>36$ weeks and birth weight $>2.4 \mathrm{~kg}$, no acute illness or pregnancy or delivery complications, and no known current maternal TB or known TB exposure in the household. At the time of this study, pregnant women were either prescribed dual therapy for prevention of MTCT or cART therapy if CD4 count was below 350 cells $/ \mathrm{mm}^{3}$. In total, 178 infants were assessed for study participation eligibility (Figure 1). Of these infants, 149 were enrolled and 29 were excluded from participation. Reasons for exclusion included maternal refusal to participate, receipt of BCG vaccination prior to randomization, and not meeting study inclusion criteria.

Neonates were enrolled within 12 hours of birth. Infant blood was obtained at enrollment, and HIV DNA PCR testing was performed. Infants returned at day 2 or 3 of life for HIV DNA PCR results, and BCG was administered intradermally to HIV DNA PCR-negative infants only who were randomized to the routine BCG arm. Thereafter, infants were followed up at 2, 6, 8, and 14 weeks of age, when a detailed medical, social, and demographic questionnaire was completed, and physical examination and phlebotomy was performed. Infants in the delayed arm were vaccinated at 8 weeks of age, provided their 6-week HIV DNA PCR was negative. Based on WHO recommendations at the time, infants testing HIV DNA PCR positive were not vaccinated with BCG and were referred immediately for cART therapy. All 
other routine infant vaccinations were administered according to the South African vaccination schedule, including oral poliovirus vaccination at birth. PBMCs at all time points were extracted from blood obtained in sodium heparin tubes at birth and 2, 6, 8, and 14 weeks of age within 6 hours and stored on liquid nitrogen $\left(\mathrm{LN}_{2}\right)$ in FCS containing $10 \%$ DMSO. Plasma and an aliquot of PBMCs in RNAProtect (Qiagen) were stored at $-80^{\circ} \mathrm{C}$.

Randomization. Infants were randomized to routine (i.e., early, before 3 days of age, per local standard of care) versus delayed (at 8 weeks of age) intradermal Danish strain BCG 1331 (Statens Serum Institut) vaccination via a statistician-generated randomization list. Sequentially numbered folders were prepopulated according to randomization arm. After randomization, neither study personnel nor participants were blinded. All laboratory assays were performed by laboratory staff who were blinded to infant randomization status.

Flow cytometric analysis. PBMCs stored on $\mathrm{LN}_{2}$ from all time points were thawed, washed, and stained in 96-well plates. PBMCs were incubated with LIVEDEAD Fixable Violet Dead Cell Stain or Aqua Dead Cell Stain (Invitrogen) for 20 minutes at room temperature and then washed twice with 1\% FCS PBS.

Measurement of $T$ cell activation. Extracellular staining was performed for 20 minutes at room temperature with PerCP-Cy5.5-labeled anti-CD4 (BD), QDot605-labeled anti-CD8 (Invitrogen), APC-labeled CCR5 (BD), PE-Cy7-labeled anti-CD38 (E-Biosciences), and PE-labeled anti-HLA-DR (BD). Cells were washed twice with 1\% FCS PBS, centrifuged, fixed, and permeabilized using BD CytoFix/CytoPerm for 20 minutes at room temperature and washed twice with Permawash (BD). Cells were then stained with Alexa Flour700-labeled anti-CD3 (BD) and FITC-labeled anti-Ki67 (BD). Samples with fewer than 1,000 live $\mathrm{CD}^{+}$events were excluded from analysis.

Measurement of monocyte and NK cell activation. Extracellular staining was performed for 20 minutes at room temperature with PE Cy5-labeled anti-CD3 and anti-CD19 (clones UCHT1 and H1B19, respectively, BD), Qdot605-labeled anti-CD14 (clone TÜK4, Life Technologies), V450-labeled anti-CD16 (clone 3G8, BD), PE-Cy7-labeled anti-CD56 (NCAM 16.2, BD), FITC-labeled anti-HLA-DR (clone G46-6, BD), APC-labeled anti-PD-1 (clone MIH4, BD), and PE-labeled anti-Tim3 (clone 344823, R\&D Systems). Cells were washed with BD Permawash, centrifuged, and fixed with BD Cell Fix.

Cell fluorescence was measured using an LSRII (BD Immunocytometry Systems), and compensation and analysis were performed using FlowJo (Tree Star Inc.). Fluorescence minus one (FMOs) were used to distinguish positive and negative cell populations. Boolean gating was used to determine cells that were coexpressing markers, such as CD4, CCR5, CD38, and HLA-DR.

Measurement of monocyte activation gene transcripts in PBMCs. PBMCs from the 2-week time point were used to measure early differences in global or innate activation that might precede $\mathrm{T}$ cell activation at 6 weeks. RNA was extracted from PBMCs stored in RNAProtect (Qiagen) at $-80^{\circ} \mathrm{C}$ using the RNAEASY kit (Qiagen). Total RNA was reverse transcribed into first-strand cDNA using a QuantiTect Reverse Transcription Kit (Qiagen). cDNA (2.5 $\mu 1)$ was mixed with TaqMan Preamp Master Mix ( $5 \mu 1$, Applied Biosystems) and $2.5 \mu \mathrm{l}$ of STA Multiplex Primer Pool. Subsequently, cDNA was preamplified using sequence-specific primers by denaturation at $95^{\circ} \mathrm{C}$ and annealing and amplification at $60^{\circ} \mathrm{C}$ for 4 minutes for 14 cycles. Primer lists for gene expression analyses are listed in Supplemental Table 1. These preamplified products were diluted 5-fold prior to analysis with Fast Start TaqMan Probe Master Mix and inventoried Taqman gene expression assays (Applied Biosystems) in 48.48 Dynamic Arrays on a Biomark System (Fluidigm). $\mathrm{Ct}$ values were calculated from the system software (Biomark Real-Time PCR Analysis, Fluidigm). The expression value of each gene relative to GAPDH was also calculated using the $\triangle \triangle \mathrm{Ct}$ methods. Both GAP$\mathrm{DH}$ and HPRT were initially selected for use as endogenous control genes. While both candidates yielded similar gene expression patterns using the $\mathrm{Ct}$ method, GAPDH alone was selected for use in the final data analysis, as its ubiquitously higher expression level permitted the inclusion of more infants in the analysis.

Measurement of plasma cytokines. Plasma samples from week 2 and week 6 were thawed on ice. Prior to cytokine measurements, plasma was centrifuged at 10,000 $\mathrm{g}$ for 10 minutes to remove debris. GMCSF, IFN- $\alpha$, IFN- $\gamma$, IL-8, IP-10, MCP-1, MIP-1 $\beta$, TNF- $\alpha$, and IL-10 concentrations in plasma samples were determined using Milliplex Human Cytokine kits (Millipore). The sensitivity of these kits ranges between 0.2 and $0.7 \mathrm{pg} /$ $\mathrm{ml}$ for the cytokines measured, and all cytokines were measured in duplicate. Data were collected using a BioPlex Suspension Array Reader (Bio-Rad Laboratories). Data were analyzed using Bio-Plex manager software (version 4; Bio-Rad Laboratories). Cytokine levels that were below the lower limit of detection of the assay were reported as the mid-point between the lowest concentrations measured for each cytokine and 0 . 
Plasma samples from the 8-week time point were thawed on ice. Prior to analyses, samples were centrifuged at 10,000 $\mathrm{g}$ for 10 minutes to remove debris. The sCD163 (R\&D Systems) ELISAs were performed according to the manufacturer's instructions. Optical densities were measured at $450 \mathrm{~nm}$, and the concentration of protein in each sample was calculated by averaging the optical density of the duplicate samples and plotting against a standard curve.

Statistics. Data analysis was based on intent to treat, i.e., randomization status and post-hoc data analysis were also performed on a per-protocol basis. Results were generally consistent between analyses. The sample size calculation was based on frequencies of $C D 4^{+} \mathrm{T}$ cells expressing HLA-DR at 6 weeks of age from a previous cohort. Using a rank-sum (Mann-Whitney $U$ ) test, a sample size of 48 infants in each arm would allow $90 \%$ power to detect a $0.5 \%$ difference in frequency (2-tailed test at $\alpha=0.05$ ). Therefore, the primary outcome measure was the HLA-DR ${ }^{+} \mathrm{CD} 4^{+} \mathrm{T}$ cell frequency at 6 weeks of age (before the delayed group received their vaccination) between infants receiving routine BCG vaccination and those receiving delayed vaccination. A $P$ value of less than 0.05 was considered statistically significant. Secondary outcomes included frequency of $\mathrm{CD} 4^{+} \mathrm{CCR} 5^{+} \mathrm{HLA}-\mathrm{DR}{ }^{+} \mathrm{CD} 38^{+} \mathrm{T}$ cells (activated HIV target cells) throughout the study period. Data were analyzed using Stata version 11 (Statcorp). Nonnormally distributed data were $\log$ transformed prior to statistical testing. Where appropriate, adjustments for multiple comparisons were performed using the Holm step-down approach. Multiple linear and nonparametric regression models were used to examine the differences in immune activation between groups after making statistical adjustments for demographic and immunological covariates. Generalized estimating equation population-averaged models were used to determine the difference between groups longitudinally.

Study approval. This study was approved by the University of Cape Town and Stellenbosch University Research ethics committees (protocols 405/2009 and N10/04/125, respectively) and the University of Washington institutional review board (protocol 38213). Written informed consent was obtained from all mothers prior to inclusion of their infants in the study.

\section{Author contributions}

HBJ, ACH, DLS, WH, JAP and MFC designed the study. HBJ, MAG, IM, and TA performed experiments. HBJ, MAG, IM, INC, and LM provided data analysis and interpretation. All authors prepared the manuscript.

\section{Acknowledgments}

This work was supported by funding from the Elizabeth Glaser Pediatric AIDS Foundation (grant MV-009-900-01871-0-00) and by a Thrasher Foundation Early Career Award to HBJ (grant NR-0095). DLS was supported by the National Institute of Dental and Craniofacial Research (DE023047). MAG was supported in part by the University of Washington Global Opportunities Fellowship, the Viral Pathogenesis training grant (NIH T32-AI083203-01), and the University of Washington Sexually Transmitted Infection Cooperative Research Center (NIH U19-AI11317). The authors thank the Western Cape Department of Health and the City of Cape Town for facilitating access to the clinic.

Address correspondence to: Heather B. Jaspan, Seattle Children's Research Institute, 1900 9th Avenue, Seattle, Washington 98101,USA. Phone: 206.987.2073; E-mail: heather.jaspan@seattlechildrens.org.

MAG and HBJ's present address is: Seattle Children's Research Institute, University of Washington, Seattle, Washington, USA.

1. UNAIDS report on the global AIDS epidemic 2013. UNAIDS. http://www.unaids.org/sites/default/files/media_asset/ UNAIDS_Global_Report_2013_en_1.pdf. Accessed March 9, 2017.

2. Trunz BB, Fine P, Dye C. Effect of BCG vaccination on childhood tuberculous meningitis and miliary tuberculosis worldwide: a meta-analysis and assessment of cost-effectiveness. Lancet. 2006;367(9517):1173-1180.

3. Fine P, Carneiro I, Milstien J, Clements C. Issues relating to the use of BCG immunization programmes-a discussion document. World Health Organization. http://apps.who.int/iris/handle/10665/66120. Accessed March 9, 2017.

4. Kidzeru EB, et al. In-utero exposure to maternal HIV infection alters T-cell immune responses to vaccination in HIV-uninfected infants. AIDS. 2014;28(10):1421-1430.

5. Van Rie A, et al. Gamma interferon production in response to Mycobacterium bovis BCG and Mycobacterium tuberculosis antigens in infants born to human immunodeficiency virus-infected mothers. Clin Vaccine Immunol. 2006;13(2):246-252. 
6. Card CM, et al. Reduced cellular susceptibility to in vitro HIV infection is associated with CD4+ T cell quiescence. PLoS ONE. 2012;7(9):e45911.

7. Bégaud E, et al. Reduced CD4 T cell activation and in vitro susceptibility to HIV-1 infection in exposed uninfected Central Africans. Retrovirology. 2006;3:35.

8. Koning FA, et al. Low-level CD4+ T cell activation is associated with low susceptibility to HIV-1 infection. J Immunol. 2005; 175(9):6117-6122.

9. Chahroudi A, et al. Target cell availability, rather than breast milk factors, dictates mother-to-infant transmission of SIV in sooty mangabeys and rhesus macaques. PLoS Pathog. 2014;10(3):e1003958.

10. Jensen $\mathrm{K}$, et al. Balancing trained immunity with persistent immune activation and the risk of simian immunodeficiency virus infection in infant macaques vaccinated with attenuated Mycobacterium tuberculosis or Mycobacterium bovis BCG vaccine. Clin Vaccine Immunol. 2017;24(1):e00360.

11. Prentice S, Webb EL, Dockrell HM, Kaleebu P, Elliott AM, Cose S. Investigating the non-specific effects of BCG vaccination on the innate immune system in Ugandan neonates: study protocol for a randomised controlled trial. Trials. 2015;16:149.

12. Kleinnijenhuis J, et al. Bacille Calmette-Guerin induces NOD2-dependent nonspecific protection from reinfection via epigenetic reprogramming of monocytes. Proc Natl Acad Sci USA. 2012;109(43):17537-17542.

13. Bryson YJ, Luzuriaga K, Sullivan JL, Wara DW. Proposed definitions for in utero versus intrapartum transmission of HIV-1. $N$ Engl J Med. 1992;327(17):1246-1247.

14. Zhang Z, et al. Sexual transmission and propagation of SIV and HIV in resting and activated CD4+ T cells. Science. 1999;286(5443):1353-1357.

15. Meditz AL, et al. HLA-DR+ CD38 + CD4+ T lymphocytes have elevated CCR5 expression and produce the majority of R5-tropic HIV-1 RNA in vivo. J Virol. 2011;85(19):10189-10200.

16. Kleinnijenhuis J, et al. BCG-induced trained immunity in NK cells: Role for non-specific protection to infection. Clin Immunol. 2014;155(2):213-219.

17. Zhang Y, et al. Tim-3 regulates pro- and anti-inflammatory cytokine expression in human CD14+ monocytes. J Leukoc Biol. 2012;91(2):189-196.

18. Alvarez IB, et al. Role played by the programmed death-1-programmed death ligand pathway during innate immunity against Mycobacterium tuberculosis. J Infect Dis. 2010;202(4):524-532.

19. Hanna J, Bechtel P, Zhai Y, Youssef F, McLachlan K, Mandelboim O. Novel insights on human NK cells' immunological modalities revealed by gene expression profiling. J Immunol. 2004;173(11):6547-6563.

20. Masson L, et al. Defining genital tract cytokine signatures of sexually transmitted infections and bacterial vaginosis in women at high risk of HIV infection: a cross-sectional study. Sex Transm Infect. 2014;90(8):580-587.

21. Fletcher HA, et al. T-cell activation is an immune correlate of risk in BCG vaccinated infants. Nat Commun. 2016;7:11290

22. Dutta A, et al. Altered T-bet dominance in IFN- $\gamma$-decoupled CD4+ T cells with attenuated cytokine storm and preserved memory in influenza. J Immunol. 2013;190(8):4205-4214.

23. Gallagher M, et al. The effects of maternal helminth and malaria infections on mother-to-child HIV transmission. AIDS. 2005;19(16):1849-1855.

24. Milush JM, et al. Rapid dissemination of SIV following oral inoculation. AIDS. 2004;18(18):2371-2380.

25. Madhi SA, et al. Primary isoniazid prophylaxis against tuberculosis in HIV-exposed children. N Engl J Med. 2011;365(1):21-31.

26. Cheynier R, Gratton S, Halloran M, Stahmer I, Letvin NL, Wain-Hobson S. Antigenic stimulation by BCG vaccine as an in vivo driving force for SIV replication and dissemination. Nat Med. 1998;4(4):421-427.

27. Zhou D, et al. Mycobacterium bovis bacille Calmette-Guérin enhances pathogenicity of simian immunodeficiency virus infection and accelerates progression to AIDS in macaques: a role of persistent T cell activation in AIDS pathogenesis. J Immunol. 1999;162(4):2204-2216.

28. Sullivan ZA, Wong EB, Ndung'u T, Kasprowicz VO, Bishai WR. Latent and active tuberculosis infection increase immune activation in individuals co-infected with HIV. EBioMedicine. 2015;2(4):334-340.

29. Brichacek B, Swindells S, Janoff EN, Pirruccello S, Stevenson M. Increased plasma human immunodeficiency virus type 1 burden following antigenic challenge with pneumococcal vaccine. J Infect Dis. 1996;174(6):1191-1199.

30. Staprans SI, et al. Activation of virus replication after vaccination of HIV-1-infected individuals. J Exp Med. 1995;182(6):1727-1737.

31. Moolasart V, et al. Long-term seroprotective response of trivalent seasonal influenza vaccine in HIV-infected children, regardless of immunogenicity before immunisation. Int J STD AIDS. 2016;27(9):761-768.

32. Lee KY, et al. Pneumococcal vaccination among HIV-infected adult patients in the era of combination antiretroviral therapy Hum Vaccin Immunother. 2014;10(12):3700-3710.

33. Hesseling AC, et al. Bacille Calmette-Guérin vaccine-induced disease in HIV-infected and HIV-uninfected children. Clin Infect Dis. 2006;42(4):548-558

34. Smith K, et al. Immune reconstitution inflammatory syndrome among HIV-infected South African infants initiating antiretroviral therapy. AIDS. 2009;23(9):1097-1107.

35. Rabie H, et al. Early antiretroviral treatment reduces risk of bacille Calmette-Guérin immune reconstitution adenitis. Int J Tuberc Lung Dis. 2011;15(9):1194-1200.

36. Aaby P, et al. Randomized trial of BCG vaccination at birth to low-birth-weight children: beneficial nonspecific effects in the neonatal period? J Infect Dis. 2011;204(2):245-252.

37. Biering-Sørensen S, et al. Small randomized trial among low-birth-weight children receiving bacillus Calmette-Guérin vaccination at first health center contact. Pediatr Infect Dis J. 2012;31(3):306-308

38. Tchakoute CT, Hesseling AC, Blakney AK, Jaspan HB. Reply to Thysen et al. J Infect Dis. 2015;212(8):1342-1343

39. Higgins JP, et al. Association of BCG, DTP, and measles containing vaccines with childhood mortality: systematic review. $B M J$. 2016;355:i5170

40. Jensen KJ, et al. Heterologous immunological effects of early BCG vaccination in low-birth-weight infants in Guinea-Bissau: a randomized-controlled trial. J Infect Dis. 2015;211(6):956-967.

41. van Crevel R, Ottenhoff TH, van der Meer JW. Innate immunity to Mycobacterium tuberculosis. Clin Microbiol Rev. 
2002;15(2):294-309

42. Porter KA, et al. CIITA enhances HIV-1 attachment to CD4+ T cells leading to enhanced infection and cell depletion. J Immunol. 2010;185(11):6480-6488.

43. Cooper MA, et al. Human natural killer cells: a unique innate immunoregulatory role for the CD56(bright) subset. Blood. 2001;97(10):3146-3151. 